\title{
Simulation of transmission performance for equalized holographic ROADM designs
}

\section{Simulación del comportamiento en transmisión para diseños de ROADMs holográficos ecualizados}

\author{
Alfredo Martin-Minguez $\left.{ }^{*}\right)$, Paloma R. Horche \\ Departamento de Tecnología Fotónica, E.T.S.I de Telecomunicación-UPM, Madrid, Spain. \\ ${ }^{(*)}$ Email: alfredo.minguez@tfo.upm.es \\ Recibido / Received: 08/02/2012. Revisado / Revised: 10/04/2012. Aceptado / Accepted: 24/04/2012.
}

\begin{abstract}
:
A complete simulation of the transmission performance for Equalized Holographic ROADM (Reconfigurable Optical Add-Drop Multiplexer) designs is presented in this paper. These devices can address several wavelengths from the input to different output fibres, according to the holograms stored in a SLM (Spatial Light Modulator), where all the outputs are equalized in power. All combinations of the input wavelengths are possible at the different output fibres. To simulate the transmission performance of the EH-ROADM, a software program, from Optiwave, has been used. The correspondence between physical blocks of the device (grating, SLM, lens...) and those simulated in the program (filters, losses, splitters...) has been defined in order to obtain a close agreement between the theoretical transmission performance and the simulated one. To complete the review about equalized holographic ROADMs some guidelines about its design have been done.
\end{abstract}

Key words: Reconfigurable Optical Add-Drop Multiplexer, Spatial Light Modulator, Coarse Wavelength Division Multiplexing, Banyan switch, Bessel filter.

\section{RESUMEN:}

En este artículo se presenta una simulación del funcionamiento en transmisión para diseños de ROADMs (Reconfigurable Optical Add-Drop Multiplexer) Holográficos Ecualizados. Estos dispositivos pueden direccionar varias longitudes de onda de las fibras de entrada a fibras de salida diferentes, según los hologramas almacenados en un SLM (Modulador Espacial de Luz) presentando, además, la potencia de salida ecualizada. Para simular el funcionamiento en transmisión del EH-ROADM, se ha utilizado un programa de software, del fabricante Optiwave, particularizado para nuestra aplicación. La correspondencia entre los bloques físicos del dispositivo (la red de difracción, el SLM, la lente,...) y aquellos simulados en el programa (filtros, pérdidas, divisores de potencia,..) se ha definido para obtener una concordancia ajustada entre el funcionamiento en transmisión teórico y el simulado. También se han incluido en el artículo algunas indicaciones sobre el diseño de ROADMs Holográficos Ecualizados, con el objeto de completar la simulación.

Palabras clave: Enrutador Óptico Reconfigurable, Modulador Espacial de Luz, Multiplexación por División en Longitud de Onda Gruesa, Conmutador de Banyan, Filtro de Bessel.

\section{REFERENCIAS Y ENLACES / REFERENCES AND LINKS}

[1]. J. Homa, K. Bala, "ROADM architectures and their enabling WSS technology", IEEE Commun. Mag. 46, 150-153 (2008).

[2]. B. Fracasso, J. L. de Bougrenet de la Tocnaye, M. Razzak, C. Uche, "Design and performance of a versatile holographic liquid-crystal wavelength-selective optical switch", J. Lightwave Technol. 21, 2405-2411 (2003). 
[3]. M. Shen, F. Xiao, S. Ahderom, K. Alameh, "An opto-VLSI-based reconfigurable optical add-drop multiplexer employing an off-axis 4-f imaging system", Opt. Express 17, 14015-14022 (2009).

[4]. A. Martín Minguez, C. del Río, P. R. Horche, "Design of equalized holographic ROADMs for applications in CWDM METRO networks", Opt. Commun. 284, 5055-5061 (2011).

[5]. S. Ahderom, M. Raisi, K. Lo, K. E. Alameh, R. Mavaddat, "Applications of liquid crystal spatial light modulators in optical communications", IEEE $5^{\text {th }}$ International Conference on High Speed Networks and Multimedia Communications 3-5, pp 239-242 (2002).

[6]. M. Dames, R. Dowling, P. McKee, D. Wood, "Efficient optical elements to generate intensity weighted spot arrays: design and fabrication", Appl. Opt. 30, 2685-2691 (1991).

[7]. M. C. Parker, A. D. Cohen, R. J. Mears, "Dynamic digital holographic wavelength filtering", J. Lightwave Techcol. 16, 1259-1270 (1998).

[8]. A. Martín Minguez, P. R. Horche, "Application of WDM holographic devices in access and metro networks", Opt. Quant. Electron. 39, 131-146 (2007).

[9]. R. Narevich, E. Narevicius, I. Vorobeichik, A. Liu, W. Long, C. Ho, H. Xu, J. Lam, "Integrated optical switch, variable attenuator and power monitor tap chip for 40-channel PLC ROADM", IEEE Photonics in Switching 2007, pp. 151 - 152 (2007).

[10]. A. Riza, S. Yuan, "Reconfigurable wavelength add-drop filtering based on a Banyan network topology and ferroelectric liquid crystal fibre optics switches", J. Lightwave Technol. 17, 1575-1584 (1999).

[11].A. D. Cohen, M. C. Parker, R. J. Mears, "100-GHz-resolution dynamic holographic channel management for WDM", IEEE Photonic. Tech. L.11, 851-853 (1999).

[12].J.-K. Rhee, F. Garcia, A. Ellis, B. Hallock, T. Kennedy, T. Lackey, R. G. Lindquist, J. P. Kondis, B. A. Scott, J. M. Harris, D. Wolf, M. Dugan, "Variable passband optical add-drop multiplexer using wavelength selective switch", $27^{\text {th }}$ European Conference on Optical Communication, 2001. ECOC '01, vol.4, pp. 550551 (2001).

[13].I. White, R. Penty, M. Webster, Y. J. Chai, A. Wonfor, S. Shahkooh, "Wavelength switching components for future photonic networks", IEEE Commun. Mag. 40, 74-81 (2002).

\section{Introduction}

For METRO, and mainly for ACCESS networks applications, an increment in capacity may be achieved with a cost-effective multiplexing technology without the need for the high number of channels and closely spaced wavelengths typically used in long haul networks with Dense Wavelength Division Multiplexing (DWDM). Coarse Wavelength Division Multiplexing (CWDM) technologies are being widely deployed internationally in metropolitan and access networks due to the increased demand for delivering more bandwidth to the subscriber, created by the need of new services. A channel space of $20 \mathrm{~nm}, \mathrm{G}$. 694.2 ITU Recommendation, can be used relaxing the processing tolerances and lowering the cost of components.

It is in this context where holographic ROADMs devices have a potential use due to their network flexibility; different technologies have been proposed for their implementation [13]. The main characteristic of holographic
ROADMs is the easy way of changing the optical wavelength and power level of the signal at the output by the dynamic implementation of different holograms on the SLM according to the requirements of network management. After the design of the device, described with detail in [4], a simulation of holographic ROADMs transmission characteristics, as a previous step to realize experimental measurements, is the goal of this work.

The organization of this paper is the following: Section 2 is dealing with a short description of the structure and main characteristics of holographic ROADMs; in Section 3 some guidelines about the design optimization of this type of devices are done; in Section 4 simulation assumptions are described whose results are shown and commented and in Section 5 a holographic router with $\lambda$ conversion and losses compensation for METRO networks application is described. 


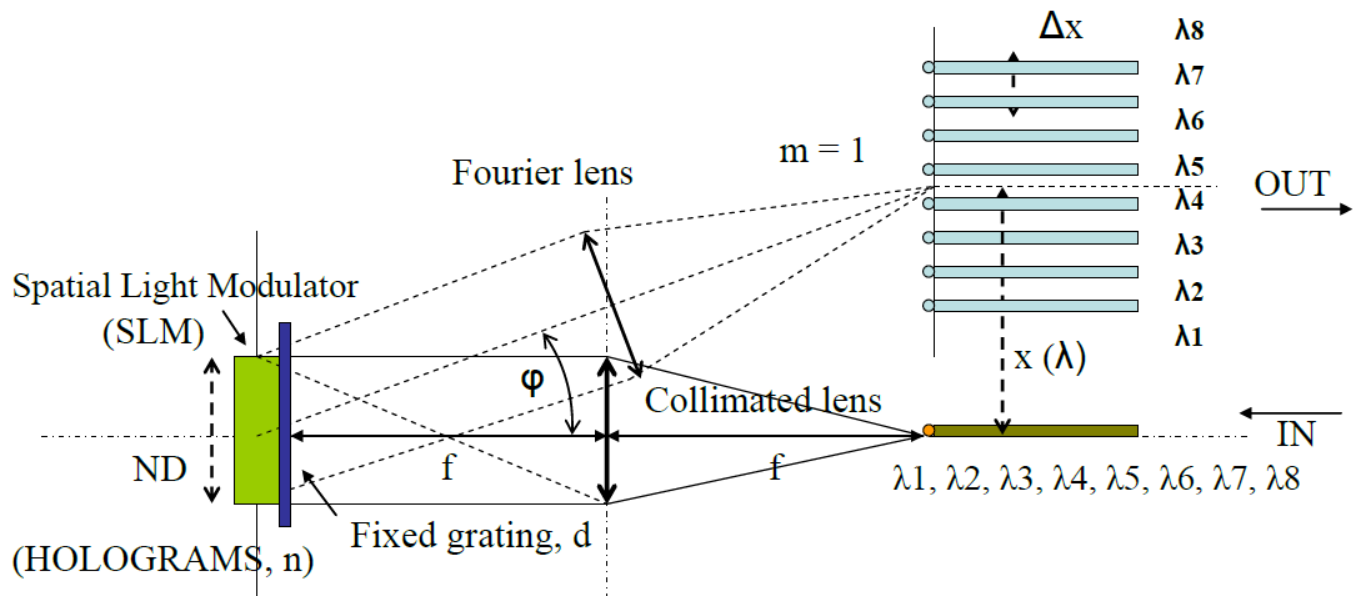

Fig. 1: Reflective holographic router.

\section{Holographic ROADM structure and characteristics}

The holographic device used in this paper for the simulation is formed by a phase reflective spatial light modulator (SLM) and a fixed transmissive diffraction grating to select the corresponding output wavelength from an ensemble of channels in the input, as it is shown in Fig. 1 [4]. The role of the fixed diffraction grating is to provide more wavelength tuning range and greater total diffraction angle. To optimize losses, 4-phase bar holograms are used with efficiency about $80 \%$ for the first order $[5,6]$. In a holographic router the tuning of the wavelength range is reached by changing the spatial period of the hologram $N D / n$, where $n$ is the number of four bars (4-phases), $N$ is the number of pixels (one dimension) and $D$ the size of the SLM pixel [7].

The losses produced in a holographic router are due to different causes:

- Diffraction loss: the total light diffraction is composed of the transmissive diffraction in the grating (twice) and the reflective diffraction in the SLM. Using 4-phases SLM and grating with first order intensity efficiency about $80 \%$, the total losses are $10 \log \left(0.8^{3}\right) \approx 3 \mathrm{~dB}$.

- Intrinsic SLM loss: it is due to the liquid crystal (LC) switching angle different to the optimal and the coverage of SLM aperture $\left(1 / e^{2}\right.$ of $N D \times$ $N D$ ). A typical value of $2 \mathrm{~dB}$ is considered.

- Fibre / lens coupling: by considering 90\% efficiency, $1 \mathrm{~dB}$ is added.
In total, with an optimized holographic device, a loss about $6 \mathrm{~dB}$ is assumed [8].

Power equalization at all output channels is necessary to compensate the different response of the network components and distances for the input channel wavelengths. To reach it, a gain component, such as a Semiconductor Optical Amplifier (SOA), together with the holographic device, has been used to obtain at the output fibres a net gain about $10 \mathrm{~dB}$. A way to have on the output selected wavelengths with different relative attenuation between them, needed for power equalization, is to control the losses due to the SLM aperture by changing the size of the hologram according to the number of active pixels.

The equalized holographic ROADM is able to select at the output fibres any combination of wavelengths at the input fibre. This operation mode is done by the selection in the SLM of a mixed hologram composed by all individual holograms corresponding to each input wavelength. Fig. 2 points out an example for three input wavelengths and its holograms, formed, in this case, by black and white bars (2phases) for simplicity.

In an equalized holographic router, the addressing of the input wavelengths at the output fibres is done by the choice of three parameters: $n_{i j}$ value, for wavelength tuning, $\mathrm{Ni}$ value for power equalization and $\Delta x_{j}$ for placing the output optical fibres (see Fig. 1). With this design philosophy, an equalized holographic 


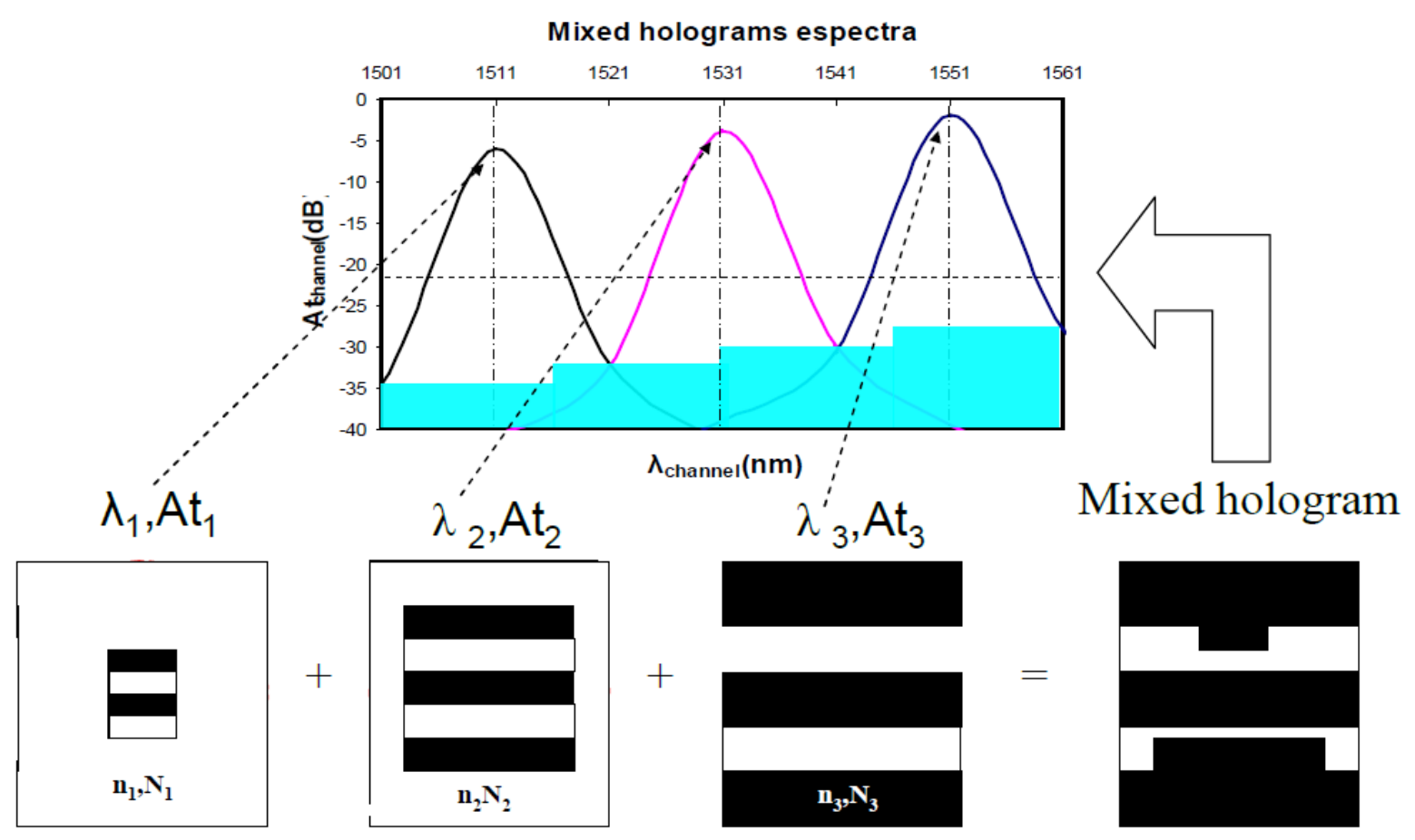

Fig. 2: Mixed holograms operation.

router can be implemented to cover a CWDM (Coarse Wavelength Division Multiplexing) 4channels grid according to the ITU G.695 Rec., for application in Access Metro networks [8].

\section{Equalized holographic ROADM optimized design}

The expression which allows the selection of the output wavelength $\lambda$, according to the physical parameters and structure of the device, is $[7,4]$ :

$$
\lambda \sim \frac{x}{f} \frac{1}{\left(\frac{n}{N D}+\frac{2}{(M / 2) d}\right)^{\prime}},
$$

where $x$ is the distance from the optical axis to the output fibre, $f$ is the focal distance of the lens, $d$ is the spatial period of the fixed diffraction grating and $M$ has into account the number of phases.

Once the SLM has been chosen, the focal distance of the lens, $f$, to illuminate with the collimated light the complete active surface $N D \times N D$ of the SLM (see Fig. 2), is related with the number of pixels $N$ and its size $D$ by the expression:

$$
f=\pi \phi_{\text {core }} \frac{N D}{4 \lambda_{0}},
$$

where $\phi_{\text {core }}$ is the input fibre core diameter and $\lambda_{0}$ the central wavelength in the operation region. The $3 \mathrm{~dB}$ passband filter bandwidth of the holographic device, BW, is:

$$
B W \geq \phi_{\text {core }} \frac{(M / 2) d}{f}\left(1-\frac{\lambda_{0}^{2}}{[(M / 2) d]^{2}}\right)^{3 / 2},
$$

if the condition $8 D \gg d$ is reached, where $d$ is the fixed grating spatial period.

In our case, we have a reflective 4-phases SLM with $N=1024$ and $D=8 \mu \mathrm{m}(N D=8.192$ $\mathrm{mm})$. Then, the focal distance for the lens is $37.655 \mathrm{~mm}$ and $B W \geq 1.52 \mathrm{~nm}(190 \mathrm{GHz})$, being $d=6.5 \mu \mathrm{m}$, the spatial period of a 4-phases transmissive diffraction grating and $\phi_{\text {core }}=9 \mu \mathrm{m}$ the core diameter of a singlemode fibre.

In Eq. (1) the selected wavelength of operation is calculated. The value of $n$ is varied from $n=0$ (for maximum wavelength) and $n=N / 4$ (for minimum wavelength); the central wavelength $\lambda_{0}$ is obtained when $n=N / 8$. For the design of a $1 \times 4$ router working in the upper band of the CWDM grid, 1471-1611 nm, we take $\lambda_{0}=1541 \mathrm{~nm}$. In this case $\lambda_{\min }=1407 \mathrm{~nm}$ and 
$\lambda_{\max }=1693 \mathrm{~nm}$; these values cover the entire CWDM upper band. The distance from the optical axis to the output fibre, (see Fig. 1), where the first order of the total diffraction is produced, is $x=9.808 \mathrm{~mm}$ and the total diffraction angle $\varphi \approx 14.6^{\circ}$.

From ITU G.694 Rec., all CWDM channels are spread $\Delta \lambda=20 \mathrm{~nm}$ to allow Direct Modulated Lasers (DML) wavelength variation in temperature and filters tolerance; therefore, an $\Delta \Lambda=(8-1) \Delta \lambda=1611-1471=140 \mathrm{~nm}$ range assumes $\Delta X=1260 \mu \mathrm{m}$, according to the relation:

$$
\Delta x \sim \Delta \lambda f\left(\frac{n}{N D}++\frac{2}{(M / 2) d}\right) .
$$

This is the maximum interval, at the output axis, where all the output fibres have to be placed. That means, a separation between fibres of $\Delta x=\Delta X /(8-1)=1260 / 7=180 \mu \mathrm{m}$.
Table I shows the holograms $\left(n_{i j}\right)$ and number of active pixels $\left(N_{i}\right)$ for a 4-channels grid according to the ITU G.695 Rec. For instance, in Fig. 1, a mixed hologram $113+95+78+61$ addresses the 4 input wavelengths $\left(\lambda_{3}+\lambda_{3}+\lambda_{5}+\lambda_{6}\right)$ to the output fibre 3 ; a mixed hologram $113+121+128+135$ addresses $\lambda_{3}$ to fibre $3, \lambda_{4}$ to fibre $4, \lambda_{5}$ to fibre 5 and $\lambda_{6}$ to fibre 6. In each case, every $\lambda_{i}$ has the corresponding $N_{i}$ range to assure the power equalization at the output fibres. Table II is a summary of the losses in the device (SOA+EHROADM) according to the different input channels. In this case, there is a net gain GT $=10$ $\mathrm{dB}$ to compensate the power variation due to different paths of the input channels along the network. The $N_{i}$ range, from 256 to 1024 , in Table I is to compensate a total of $10+2=12 \mathrm{~dB}$ of attenuation; with a step of $\Delta N_{i}=16$ the ripple at the output fibres is $<0.5 \mathrm{~dB}$.

Table I

Holograms and active pixels for an EH-ROADM $1 \times 4$.

\begin{tabular}{|c|c|c|c|c|c|}
\hline & \multicolumn{4}{|c|}{$n_{i j}($ holograms) } & \\
\hline & $\lambda_{3}=1511 \mathrm{~nm}$ & $\lambda_{4}=1531 \mathrm{~nm}$ & $\lambda_{5}=1551 \mathrm{~nm}$ & $\lambda_{6}=1571 \mathrm{~nm}$ & $\mathrm{x}_{\mathrm{j}}(\mu \mathrm{m})$ \\
\hline Fibre 3 & 113 & 95 & 78 & 61 & 9538 \\
\hline Fibre 4 & 139 & 121 & 103 & 85 & 9718 \\
\hline Fibre 5 & 165 & 146 & 128 & 110 & 9898 \\
\hline Fibre 6 & 191 & 172 & 153 & 135 & 10078 \\
\hline$N_{i}$ (pixels) & $256 \div 1024$ & $256 \div 1024$ & $256 \div 1024$ & $256 \div 1024$ & \\
\hline
\end{tabular}

Table II

SOA gain, EH-ROADM losses and total net gain.

\begin{tabular}{|c|c|c|c|}
\hline Intrinsic router loss (dB) & 6 & & $L_{H R}$ \\
\hline $\begin{array}{c}\text { Additional mixed hologram } \\
\text { loss (dB) }\end{array}$ & 6 & & $\begin{array}{c}\boldsymbol{\Delta} \boldsymbol{L}_{H R} \\
{[10 \times \log (4)]}\end{array}$ \\
\hline Max. gain of $S O A, G_{A}(\mathrm{~dB})$ & 24 & & @ 1531 nm \\
\hline \multirow[t]{2}{*}{ Min. gain of SOA, $G_{A}(\mathrm{~dB})$} & 22 & & @ $1571 \mathrm{~nm}$ \\
\hline & $\Delta G_{A}(\mathrm{dI}$ & Total loss(dB) & \\
\hline $1511 \mathrm{~nm}$ & 1.5 & 13.5 & \\
\hline $1531 \mathrm{~nm}$ & 2 & 14 & \\
\hline $1551 \mathrm{~nm}$ & 1 & 13 & \\
\hline $1571 \mathrm{~nm}$ & 0 & 12 & \\
\hline Total min. net gain, $G_{T}(\mathrm{~dB})$ & & 10 & \\
\hline
\end{tabular}




\section{Equalized holographic ROADM simulation}

After these preliminary considerations about structure and design of the device, we are going to its simulation. The physical structure of the ROADM is pointed out in Fig. 1, and the corresponding block diagram for simulation, from a transmission point of view, is shown in Fig. 3.

For every input wavelength (channel) a hologram value, ni (spatial period), is assigned, and a tuned wavelength, $\lambda_{i}, \quad(i=1,2,3,4)$ is obtained at the output. In addition, $N_{i}$ takes into account the number of active pixels to reach the correct attenuation to equalize the output signal by a variation of the SLM aperture size. This mixed hologram produces an additional loss in the holographic router, $10 \times \log$ ( $\mathrm{n}^{\mathrm{o}}$ channels). A more fitted equalization can be obtained by monitoring the outputs with a feed-back loop to adjust the size of the holograms according to the wanted output signal level.

Equalization in ROADMs is an important topic to be reached. The optical power of the input wavelengths is not the same due to the different paths through the network that they have used; also, losses of the node filtering elements impact on the channels (wavelengths) output power. A non equalized output could limit the maximum distance to cover in the optical network for a fixed quality. The roll of the SOA is to supply a net gain to the device in order to compensate the intrinsic losses and to allow the output equalization with a power balance, from the input to the outputs, next to $10 \mathrm{~dB}$. Typical gain of these devices for a bandwidth of $100 \mathrm{~nm}$ is $20-25 \mathrm{~dB}$, with a "ripple" of 4-5 dB.

The correspondence between the physical blocks of the Fig. 1 and the simulated ones of the Fig. 3, is the following:

- SLM and fixed grating assembly: $3^{\text {rd }}$ order optical Bessel filters for tuning the output wavelengths, $\lambda_{i}$.

- Lens and holograms: splitting and switching elements to select the correct hologram, $n_{i}$.

- Device fixed losses: are distributed among the different blocks: splitting (4 wavelengths) and switching elements, $<6+4<10 \mathrm{~dB}$ and filter losses, $<2 \mathrm{~dB}$. Total fixed losses, $<12 \mathrm{~dB}$.

\section{EH-ROADM 1x4: transmission simulated scheme}

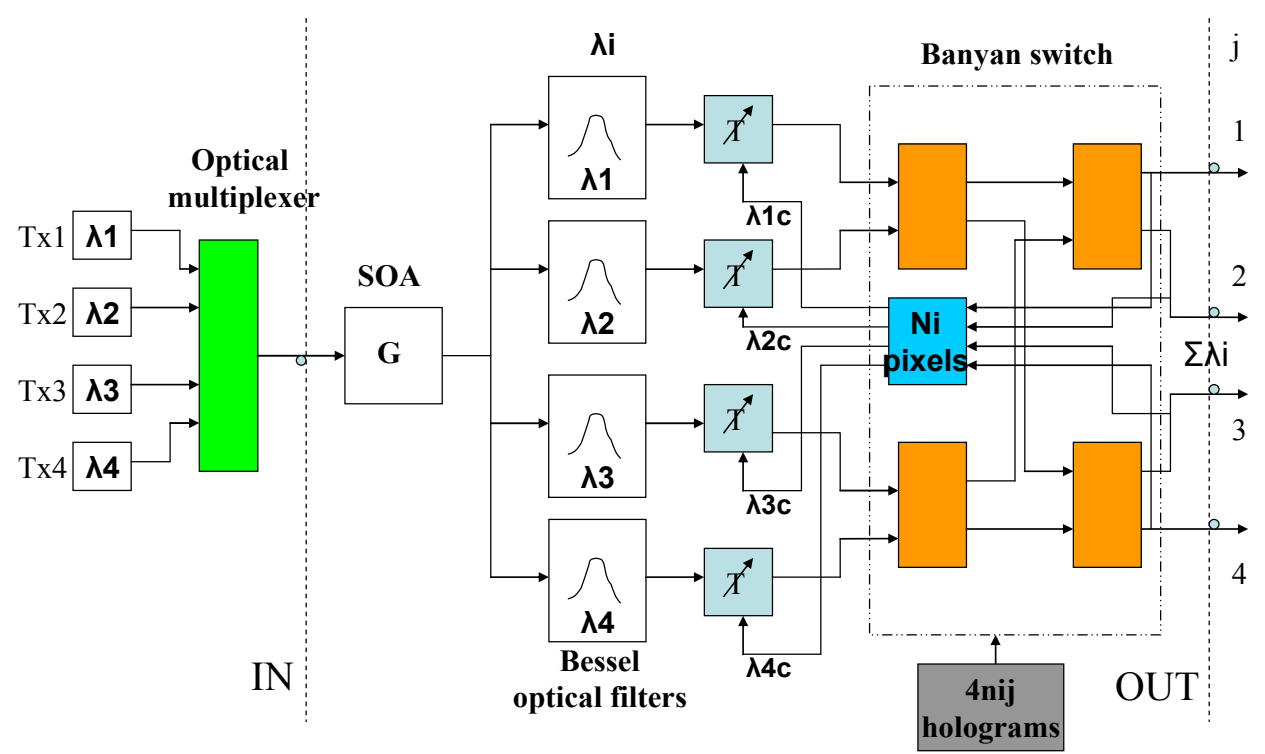

Fig. 3: Transmission simulated scheme for an equalized holographic ROADM. 


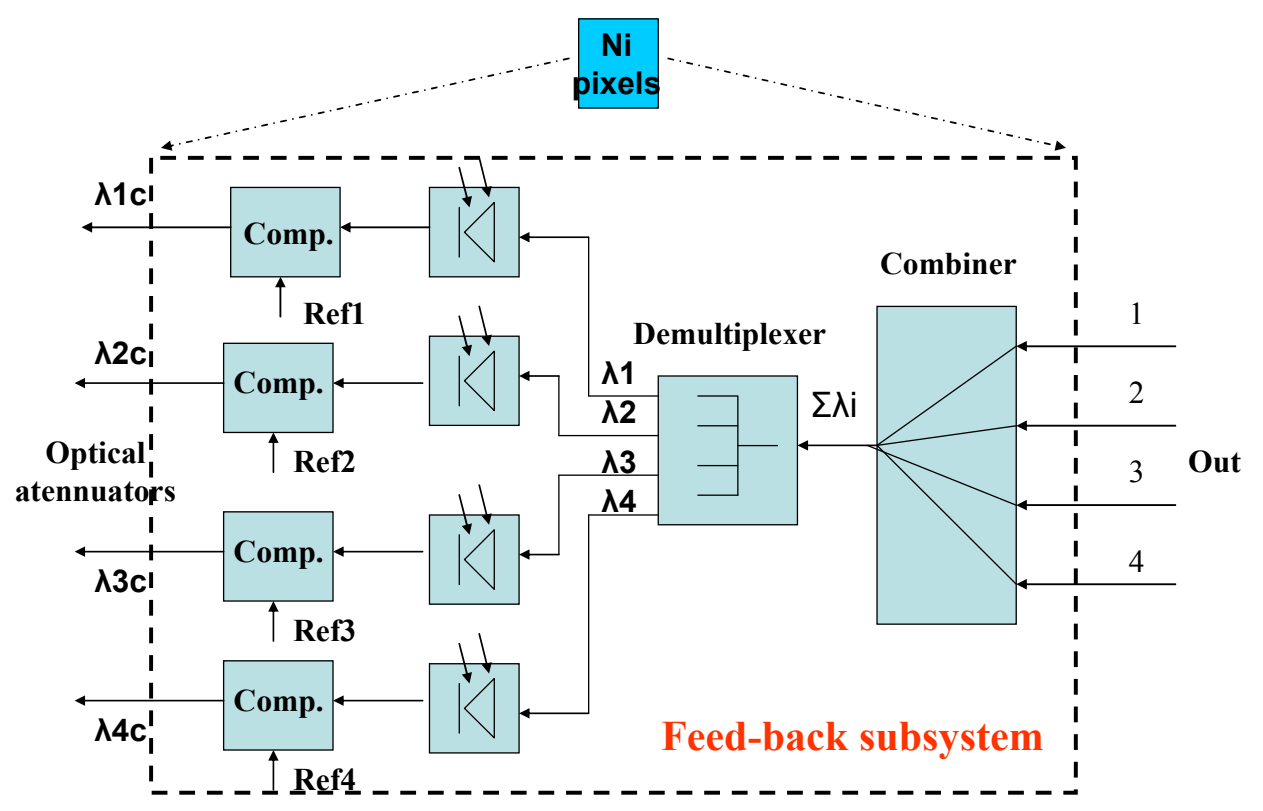

Fig. 4: Feed-back control scheme for the output signal equalization.

- Variable losses: they are due to different SLM apertures according to the input power level of the wavelengths. They are variable between 0 and $10 \mathrm{~dB}$. The diagram of the feed-back circuit to reach this equalization is shown in Fig. 4. By a demultiplexation of the output wavelengths, its power level is obtained after a detection and conversion to an electrical signal to change the attenuation of each wavelength according to a reference signal [1,9].

Complete wavelength switching $(4 \times 4)$ from the input to the output fibres is simulated by a Banyan network composed by 4 binary cells $(2 \times 2)[10]$. The internal structure of these cells is shown in Fig. 6; in this case, in both outputs, the wavelength combinations are: $\lambda_{1}, \lambda_{2}, \lambda_{1}+\lambda_{2}, 0$, obtained by the selection of the control bits $(a, b$, c, d, e, f). For the complete Banyan switch $(4 \times 4)$ the total wavelength combinations at the outputs is $64(4 \times 24)$, obtained by 64 combinations of 4 nij hologram groups. Fig. 5 shows a simulation of the wavelength response of the holographic device filter $[11,12]$.

For wavelengths close to the central, the filter response is very similar to the Gauss filter (5); for wavelengths far from the central, the filter response is similar to the $2^{\text {nd }}-3^{r d}$ order Bessel filter (6) with less out band attenuation. Both of them have a lineal phase characteristic, which means, a constant group delay. These simulations are in agreement with experimental measurements [7].

In case of a Bessel filter, the $B W$ (filter $-3 \mathrm{~dB}$ bandwidth) and out-band attenuation are reached by the increment of the filter order or the variation of the polynomial coefficients. For a Gauss filter these values are obtained, directly, by the change of the standard deviation $\sigma=B W$ :

$$
H f_{\text {Gauss }}=\frac{1}{\sqrt{2 \pi} B W} e^{-\frac{\left(\lambda-\lambda_{0}\right)^{2}}{2 B W^{2}}}
$$

with $\lambda_{0}-2 B W<\lambda<\lambda_{0}+2 B W$, and

$$
H f_{\text {Bessel }}=\frac{3}{\left(\lambda-\lambda_{0}\right)^{2}+3\left(\lambda-\lambda_{0}\right)+3} \text {. }
$$

To simulate the transmission performance of the EH-ROADM, a software program, OptiSystem from Optiwave, has been chosen. The complete

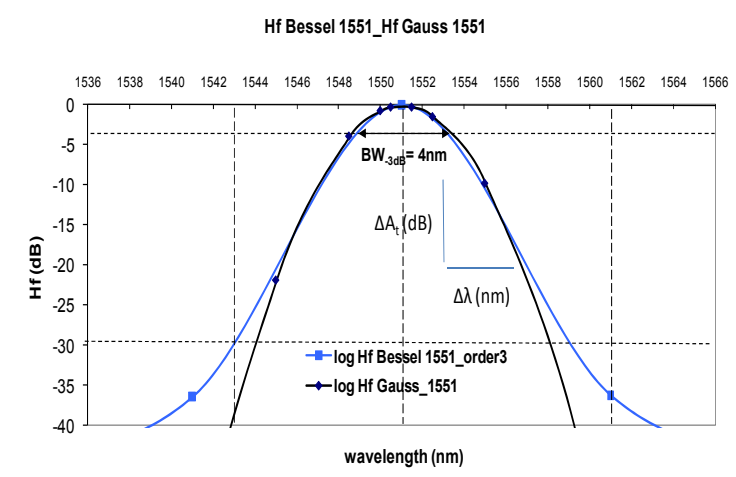

Fig. 5. Simulated response of the holographic filter device. 


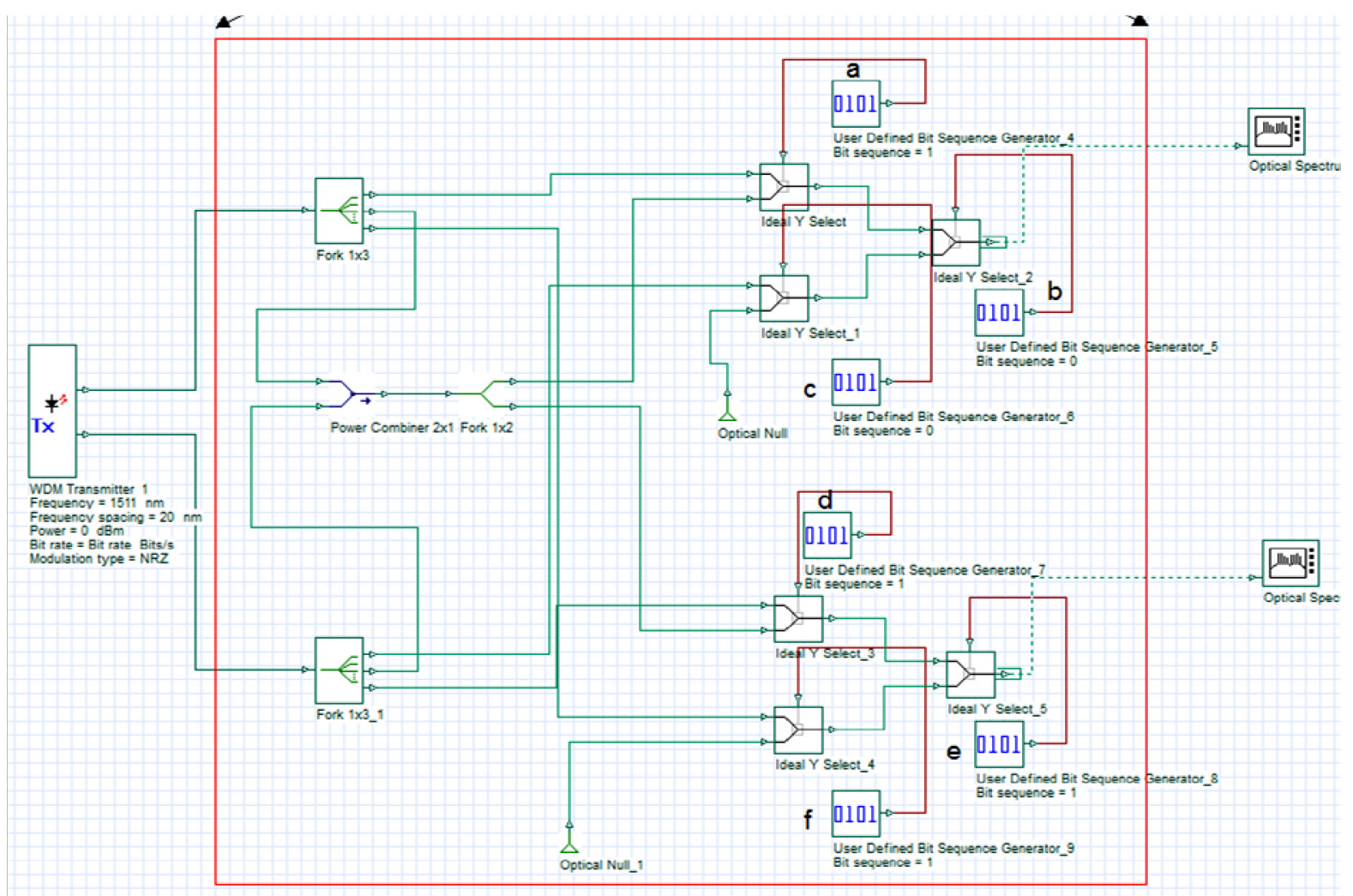

Fig. 6: Simulated binary switch of the holographic device.

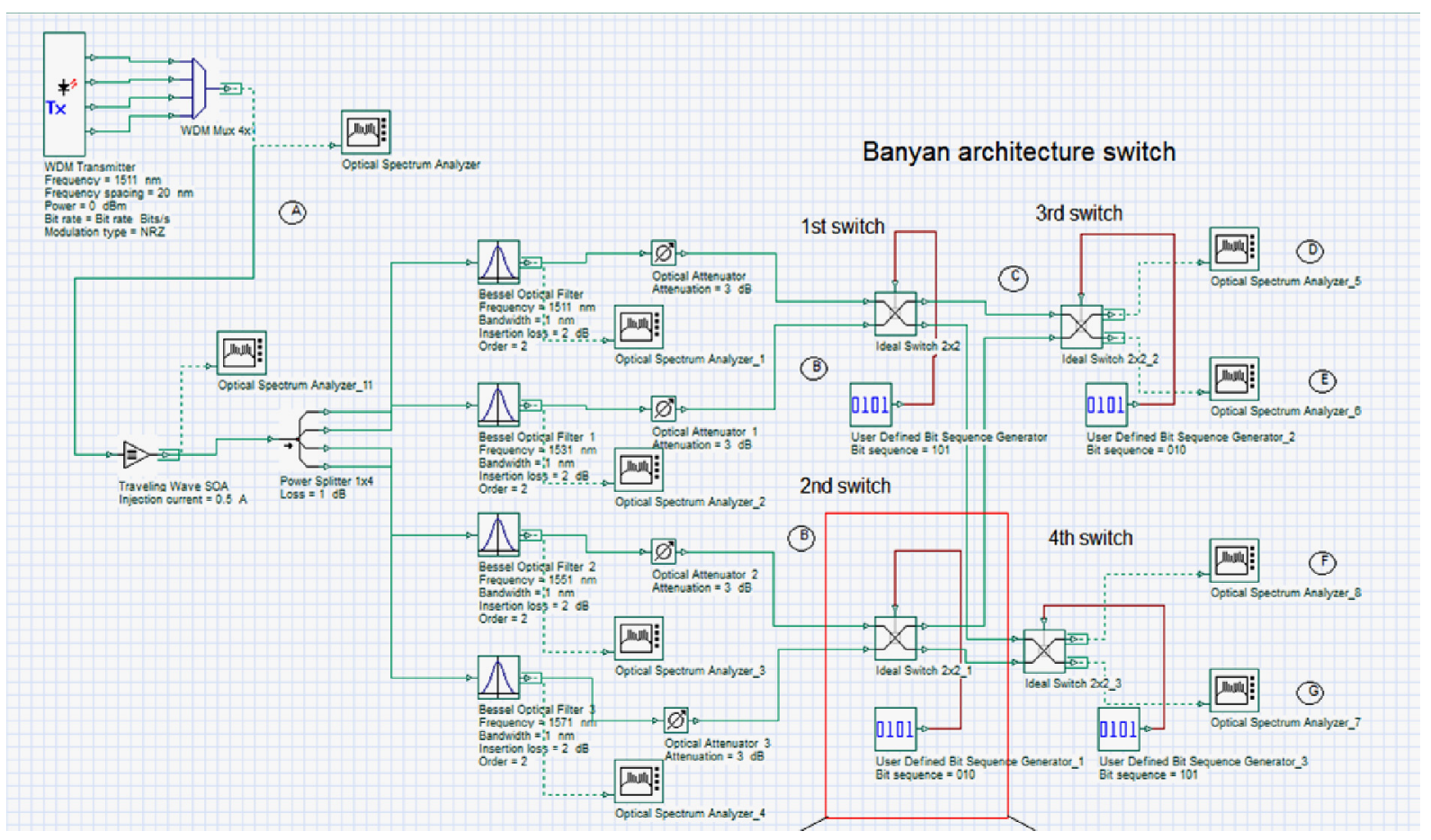

Fig. 7: Scheme for the simulation of the holographic device operation. 
set up is shown in Fig. 7. The input channels are simulated by a WDM transmitter, whose output levels can be changed to take into account the different attenuation paths along the network. To know the value of these power levels, different optical spectrum check points are available on the diagram. Bessel filters are selected at each input wavelength with the corresponding variable attenuation to reach the equalized outputs.

In Table III the outputs at the Bessel filters for channel 2 and 3 are obtained (intermediate point B). More than $40 \mathrm{~dB}$ of attenuation are reached between channel 2 (or 3) and the nearest wavelength.

In Table IV, several results obtained with three different cases are shown:

- EH-ROADM 1: $\lambda_{1}, \lambda_{2}, \lambda_{3}, \lambda_{4}$ output channels are available in output 1 .

- EH-ROADM 2: $\lambda_{1}, \lambda_{2}$ output channels are available in output 1 and $\lambda_{3}, \lambda_{4}$ in output 4 .

- EH-ROADM 3: $\lambda_{1}, \lambda_{2}, \lambda_{3}$ output channels are available in output 1 and $\lambda_{4}$ in output 4 .
In all cases, input channels have $10 \mathrm{~dB}$ of maximum power difference $(-3 \div-13 \mathrm{dBm})$ between them and the output power ripple is less than $0.5 \mathrm{~dB}(-2.7 \div-3.2 \mathrm{dBm})$. Also switching tables for the Banyan networks and the attenuation needed for output equalization, depending on the wavelength, have been calculated.

\section{Holographic router with $\lambda$ conversion and losses compensation for METRO networks}

As an example of application, Fig. 8 shows the structure of a holographic router with losses compensation and wavelength conversion, whose main application is in the interconnection nodes of Metro-Access networks. This device uses a SOA, in the non-linear region, to do the wavelength conversion and, in addition, to provide the gain in order to compensate the intrinsic losses of the holographic router and to perform the output equalization.

Table III

Signals at the intermediate point B: Output filter channels 2 and 3.

\section{EH-ROADM 1}

Input (A): Output 1x4 Mux

Power: $-3 \div-13 \mathrm{dBm}$ (input channels with $10 \mathrm{~dB}$ of power difference)

Intermediate (B): Output Bessel filter channel 2) and 3)
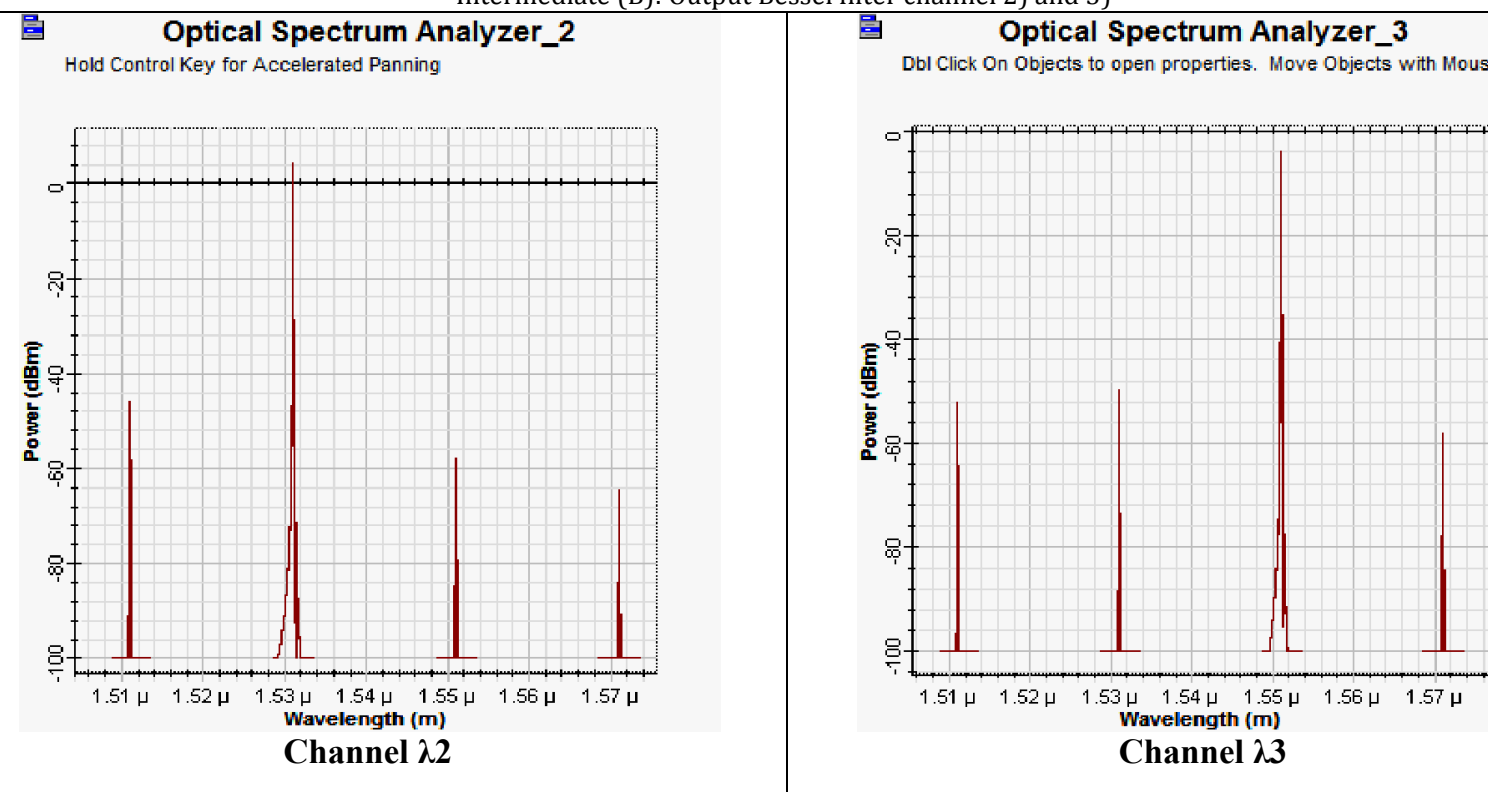
Table IV

a) $\lambda_{1}, \lambda_{2}, \lambda_{3}, \lambda_{4}$ channels in output 1 ; b) $\lambda_{1}, \lambda_{2}$ channels in output 1 and $\lambda_{3}, \lambda_{4}$ in output 4 ; c) $\lambda_{1}, \lambda_{2}, \lambda_{3}$ channels in output 1 and $\lambda_{4}$ in output 4.

\section{$\lambda 1, \lambda 2, \lambda 3, \lambda 4$ input channels with $10 \mathrm{~dB}$ of power difference: $-3 \div-13 \mathrm{dBm}$}

A) EH-ROADM 1

$\lambda 1, \lambda 2, \lambda 3, \lambda 4$ output channels are available in output 1

Output 1 (D) ----> Power: $-2.7 \div-3.1 \mathrm{dBm}$

\begin{tabular}{|c|c|c|c|c|c|c|c|}
\hline & \multicolumn{7}{|c|}{ Control } \\
\hline \multirow{5}{*}{$\begin{array}{l}\text { Bany } \\
\text { an } \\
\text { Switc } \\
\text { h }\end{array}$} & & $\mathbf{a}$ & & c & & $\mathbf{e}$ & f \\
\hline & 1 & 1 & 0 & 0 & 1 & 1 & 1 \\
\hline & 2 & 1 & 0 & 0 & 1 & 1 & 1 \\
\hline & 3 & 1 & 0 & 0 & 1 & 1 & 1 \\
\hline & 4 & 1 & 0 & 0 & 1 & 1 & 1 \\
\hline
\end{tabular}

\begin{tabular}{|c|c|c|c|c|}
\hline & $\boldsymbol{\lambda} \mathbf{1}$ & $\boldsymbol{\lambda} \mathbf{2}$ & $\boldsymbol{\lambda} \mathbf{3}$ & $\boldsymbol{\lambda}$ \\
\hline Att (dB) & 9 & 7 & 3 & 0 \\
\hline
\end{tabular}

All channels are available in output 1 with a power ripple $<0.5 \mathrm{~dB}$

\section{B) EH-ROADM 2}

$\lambda 1, \lambda 2$ output channels are available in output 1 and $\lambda 3, \lambda 4$ in output 4

Output 1 (D) -----> Power: $-2.8 \div-3.2 \mathrm{dBm}$ Output 4 (G) -----> Power: $-2.8 \div-3.2 \mathrm{dbm}$

\begin{tabular}{|c|c|c|c|c|c|c|c|}
\hline & \multicolumn{7}{|c|}{ Control } \\
\hline \multirow{4}{*}{$\begin{array}{c}\text { Banyan } \\
\text { Switch }\end{array}$} & & $\mathbf{a}$ & $\mathbf{b}$ & $\mathbf{c}$ & $\mathbf{d}$ & $\mathbf{e}$ & $\mathbf{f}$ \\
\cline { 2 - 8 } & $\mathbf{1}$ & 1 & 0 & 1 & 1 & 1 & 1 \\
\cline { 2 - 8 } & $\mathbf{2}$ & 0 & 0 & 1 & 1 & 0 & 1 \\
\cline { 2 - 8 } & $\mathbf{3}$ & 0 & 0 & 1 & 1 & 1 & 1 \\
\cline { 2 - 8 } & $\mathbf{4}$ & 1 & 1 & 1 & 0 & 0 & 1 \\
\hline
\end{tabular}

\begin{tabular}{|l|c|c|c|c|}
\hline & $\boldsymbol{\lambda} \mathbf{1}$ & $\boldsymbol{\lambda} \mathbf{2}$ & $\boldsymbol{\lambda} \mathbf{3}$ & $\boldsymbol{\lambda} \mathbf{4}$ \\
\hline Att (dB) & 12 & 10 & 6 & 3 \\
\hline
\end{tabular}

$\lambda 1, \lambda 2$ channels are available in output 1 and $\lambda 3, \lambda 4$ in output 4

\section{C) EH-ROADM 3}

$\lambda 1, \lambda 2, \lambda 3$ output channels are available in output 1 and $\lambda 4$ in output 4

Output 1 (D) -----> Power: $-2.8 \div-3.2 \mathrm{dBm}$ Output 4 (G) -----> Power: $-2.8 \div-3.2 \mathrm{dbm}$

\begin{tabular}{|c|c|c|c|c|c|c|c|}
\hline & \multicolumn{7}{|c|}{ Control } \\
\hline \multirow{4}{*}{$\begin{array}{c}\text { Banyan } \\
\text { Switch }\end{array}$} & & $\mathbf{a}$ & $\mathbf{b}$ & $\mathbf{c}$ & $\mathbf{d}$ & $\mathbf{e}$ & $\mathbf{f}$ \\
\cline { 2 - 8 } & $\mathbf{1}$ & 1 & 0 & 1 & 1 & 1 & 1 \\
\cline { 2 - 8 } & $\mathbf{2}$ & 0 & 0 & 1 & 0 & 0 & 1 \\
\cline { 2 - 8 } & $\mathbf{3}$ & 1 & 0 & 0 & 1 & 1 & 1 \\
\cline { 2 - 8 } & $\mathbf{4}$ & 0 & 0 & 0 & 0 & 0 & 1 \\
\hline
\end{tabular}

\begin{tabular}{|c|c|c|c|c|}
\hline & $\boldsymbol{\lambda} \mathbf{1}$ & $\boldsymbol{\lambda} \mathbf{2}$ & $\boldsymbol{\lambda} \mathbf{3}$ & $\boldsymbol{\lambda} \mathbf{4}$ \\
\hline Att (dB) & 9 & 7 & 6 & 6 \\
\hline
\end{tabular}

$\lambda 1, \lambda 2, \lambda 3$ channels are available in output 1 and $\lambda 4$ in output 4

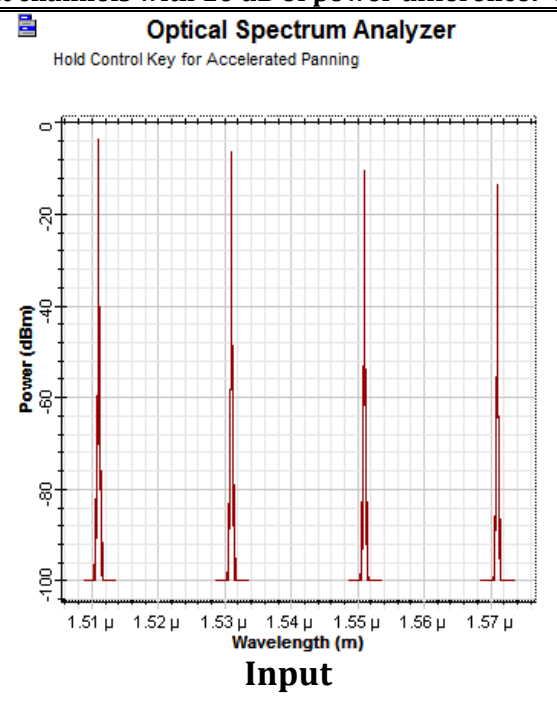

曷

Optical Spectrum Analyzer_7 ey for Accelerated Panning

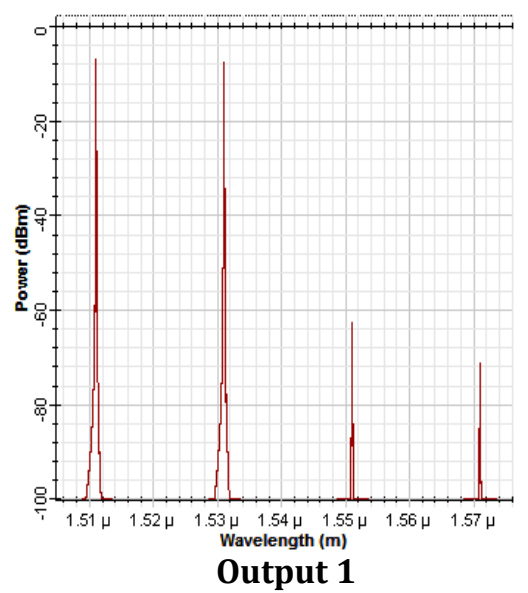

兽 Optical Spectrum Analyzer_7 Dbl cick On Objects to open properties. Move Objects with Mouse Drag

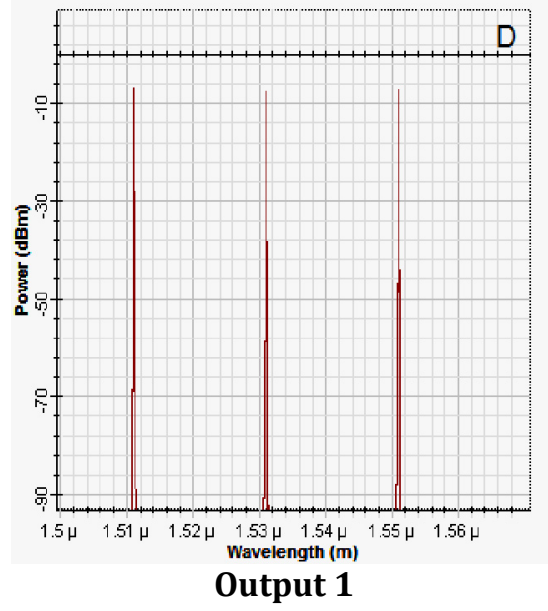

兽 Optical Spectrum Analyzer_7

Hold Control Key for Accelerated Panning

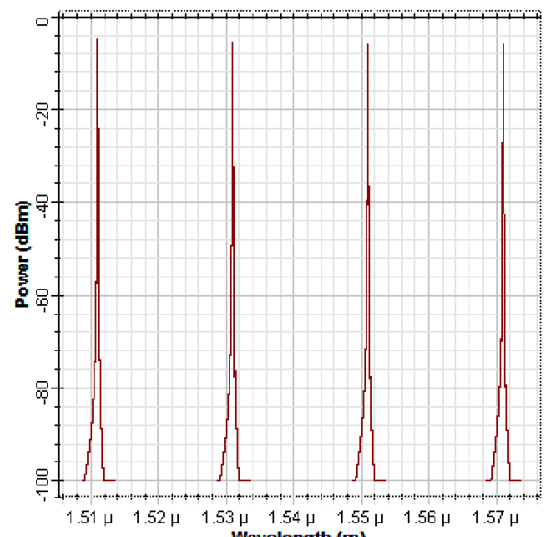

Output 1

\section{믐 Optical Spectrum Analyzer_12}

Hold Control Key and Left Mouse Button To Place Marker. Hold Shift For H

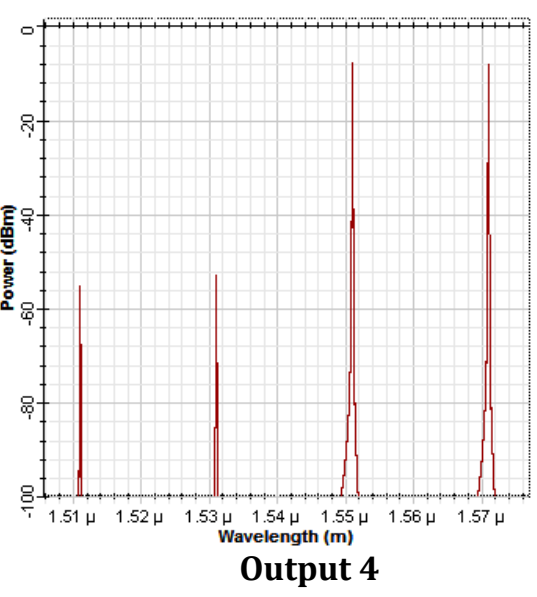

兽

Optical Spectrum Analyzer_12

Hold Control Key for Accelerated Panning

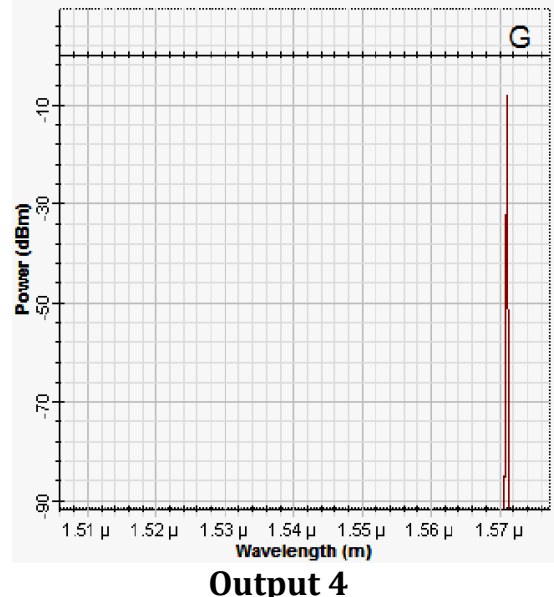




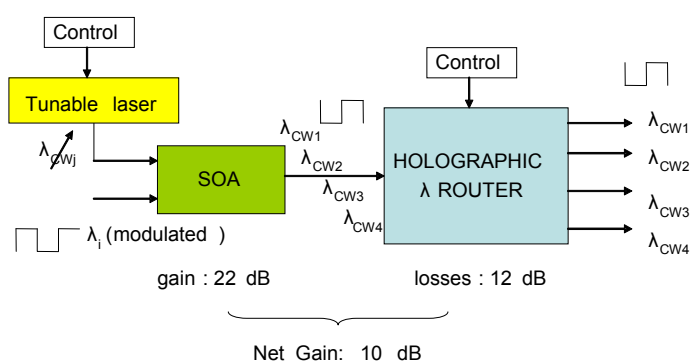

Fig. 8: Device composed of an optical $\lambda$ converter and a holographic $\lambda$ router.

The SOA performs the wavelength conversion by a non linear operation (saturation zone) using the Cross Gain Modulation (XGM) method [13]. An incident wavelength, $\lambda_{i}$, modulated by a digital signal is combined with the wavelength $\lambda_{C W j}$ generated by a tunable laser (CW) into the SOA. At the amplifier output, different $\lambda_{C W j}$ signals are obtained modulated with the digital signal from the incident $\lambda \mathrm{i}$ wavelength. These $\lambda_{C W_{j}}$ signals are also amplified and inverted.

The holographic wavelength router, depending on the input signal, $\lambda_{C W j}$, and the generated holograms $\left(n_{i j}\right)$ stored in the SLM, addresses this signal to the assigned output. As has been stated, this technology has the drawback of high insertion losses (less than 10 $\mathrm{dB}$, using an optimized device). In order to solve this problem, by combining a SOA with the holographic router, this insertion loss is compensated with the amplifier gain. A parameter to control in the SOA operation, is related with the amplified spontaneous emission (ASE) because of the impact on the signal distortion.

Figure 9 shows the simulation of the Wavelength Conversion and Routing Holographic Device (WCR-HD), composed of three different blocks:

- CW tunable laser: The type used in the simulation is a SG-DBR (Sampled GratingDistributed Bragg Reflector) laser with a range of wavelength tuning from 1520 to $1580 \mathrm{~nm}$ and an output optical power of -6 to $0 \mathrm{dBm}$. We can select the operation wavelength by an optical switch/combiner from an "array" of CW tunable lasers.

- Wavelength conversion semiconductor optical amplifier: The type used is a "quantum dot" SOA whose main advantages are: high bandwidth $(>60 \mathrm{~nm})$, low noise figure $(<5 \mathrm{~dB})$, gain $>20 \mathrm{~dB}$ and a gain recovery time in the saturation zone $<10 \mathrm{ps}$. These characteristics allow the use of the SOA for signals with bit rates up to $10 \mathrm{GBit} / \mathrm{s}$.

- Wavelength holographic router. The simulation of this device has been explained in the former section. In this example a simple operation mode has been chosen: routing of up to 4 different wavelengths (depending on the tunable laser wavelength) at the input fiber to 4 output fibers, according to the hologram value.

In Fig. 10, the response of the WCR-HD is pointed out. In this case a NRZ $2.5 \mathrm{~Gb} / \mathrm{s}$ input signal has been used, whose $\lambda_{i}=1540 \mathrm{~nm}$ is converted to an output signal, $\lambda_{0}=1520 \mathrm{~nm}$; the losses of the holographic router are compensated by the gain of the SOA, whose value is fitted by the injection current $(150 \mathrm{~mA}$, in this case). The choice of this value is a compromise between gain and distortion. A Q factor value of 100 and a very low BER are obtained.

\section{Conclusions}

The simulation of equalized holographic ROADM devices for applications in CWDM optical networks has been done with a commercial program, OptiSystem from Optiwave. These devices by using a mixed hologram, corresponding to the combination of several input wavelengths, the tuning of a broad range of wavelengths is obtained allowing the full routing of several channels from the input fibre to the outputs. As it is possible to change the active pixels in the SLM for each hologram, in order to maintain a fixed output power level, channel equalization has been reached. These characteristics have been simulated by a 


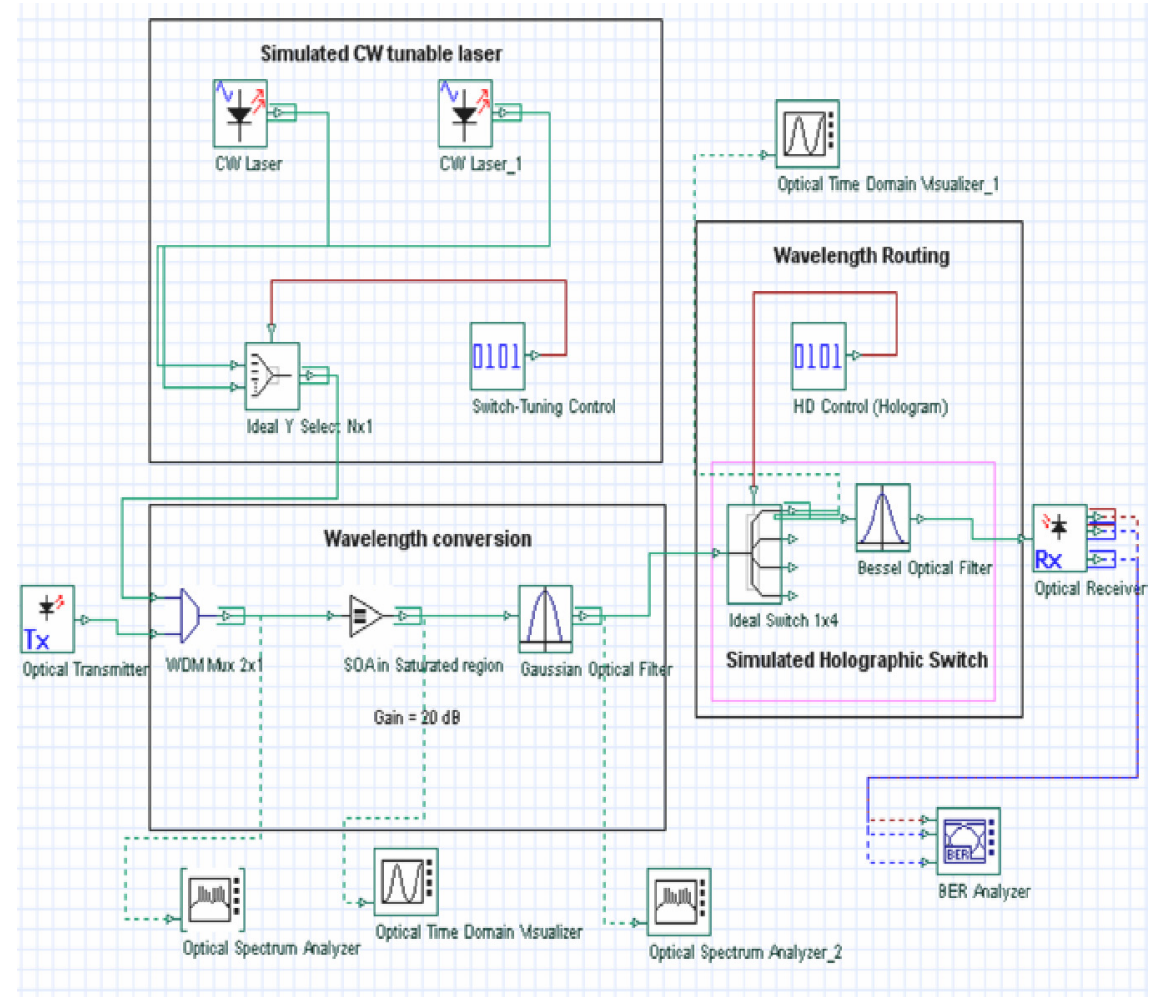

Fig. 9: Wavelength Conversion and Routing Holographic Device (WCR-HD) simulation

a) Signal Power

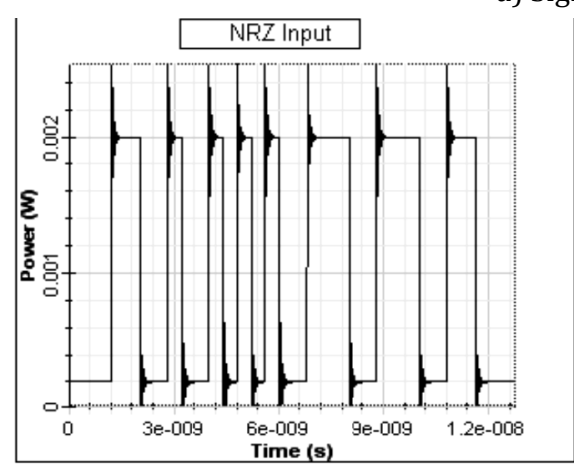

b) Q Factor

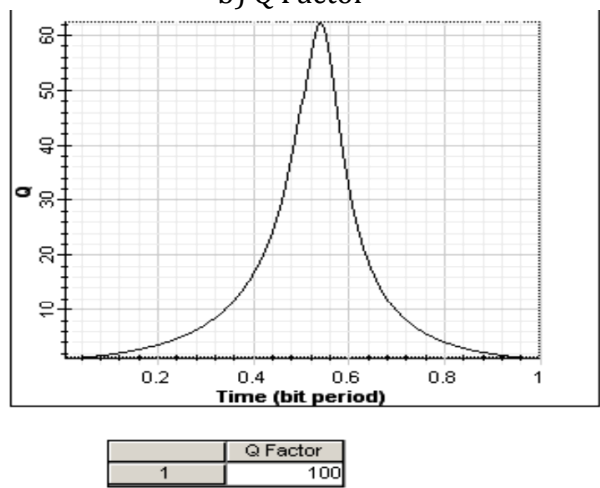

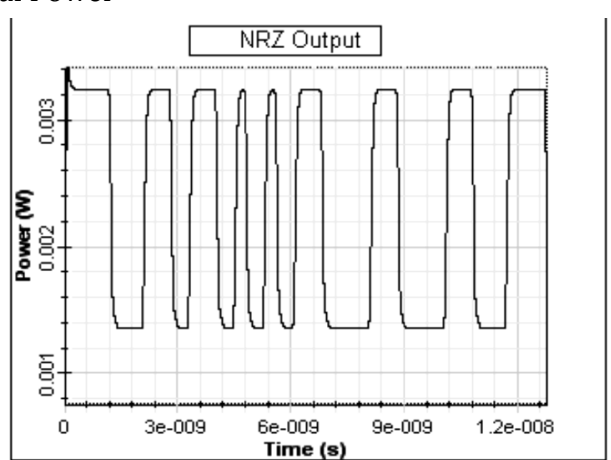

c) Eye Diagram

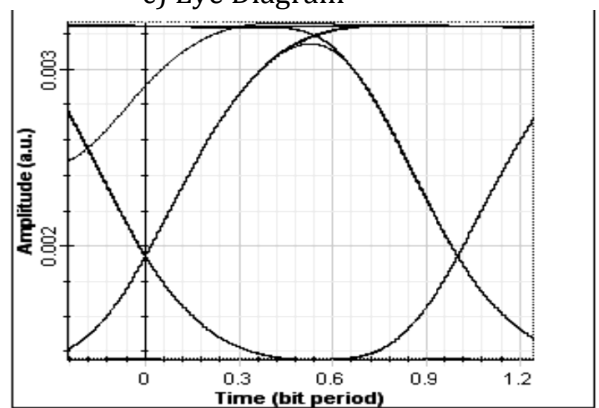

Min. BER

Fig. 10 WCR-HD response for a $2.5 \mathrm{Gbit} / \mathrm{s}$ input signal: a) $\lambda_{i}=1540 \mathrm{~nm}$, with wavelength conversion $\lambda_{0}=1520 \mathrm{~nm}$, and losses compensation, b) $\mathrm{Q}$ factor $\approx 100$ and c) BER $\approx 0$. 
correlation between the physical parameters of the device, such as lens, SLM, fixed grating,losses,..., and the simulated blocks, from the transmission point of view, such as splitters, filters, attenuations, ...

Equalization topics have been addressed by the inclusion in the ROADM design of a feed-back circuit to take into account the supervision of the optical output power for the different wavelengths. Internal switching has been simulated using a Banyan network and for filtering a Bessel approach has been chosen due to similarity with the actual response of the holographic device. Results obtained have demonstrated the validity of the simulations regarding to the assumptions made in the ROADM design based on the holographic technology for the use in CWDM networks.

\section{Acknowledgments}

The authors gratefully acknowledge the support of the MICINN (Spain) through project TEC201018540 (ROADtoPON). 